\title{
Arterial thromboembolism: Thoracic outlet syndrome complications
}

\author{
Abraham Ziga-Martinez ${ }^{1 *}$, Enrique Ortiz-Herrasti², Raúl Bacelis-Arzapalo² , Pedro Cordova-Quintal2, \\ Rogerio Muñoz-Vigna ${ }^{1}$ and Nicolás Blum-Gilbert ${ }^{1}$ \\ ${ }^{1}$ Servicio de Angiología y Cirugía Vascular, Hospital General de México, Mexico City; ${ }^{2}$ Servicio de Angiología y Cirugía Vascular, Hospital Regional \\ de Alta Especialidad de la Península de Yucatán, Mérida, Yucatán. Mexico
}

\begin{abstract}
Introduction: Thoracic outlet syndrome (TOS) results in the compression of neurovascular structures (brachial plexus, subclavian artery, and vein) between the scalene muscles and first rib. Compression of these structures can result in neurogenic and vascular TOS (arterial and venous). Case Report: We present the case of a 40-year-old female patient with acute upper limb ischemia secondary to arterial TOS. Discussion: Arterial TOS is a rare cause of ischemia of the upper limbs. It is the least common form of TOS, which represents $<1 \%$ of TOS cases. However, arterial TOS is potentially dangerous, as it can lead to acute ischemia of the upper extremities, damage of the intima, and aneurysmal degeneration of the subclavian artery related to chronic compression. Knowledge of the arterial TOS is crucial to reach a timely diagnosis and management.
\end{abstract}

Key words: Arterial thoracic outlet syndrome. Thoracic operculum. Cervical rib. Subclavian artery thrombosis. Subclavian artery aneurysm.

\section{Introduction}

In 1958, Charles Rob defined thoracic outlet syndrome (TOS) as "a set of symptoms that may exist due to compression on the brachial plexus and on subclavian vessels in the region of the thoracic outlet." TOS affects approximately $8 \%$ of the general population ${ }^{1}$. Patients often report pain, which can lead to significant disability. Weakness is another common symptom. Vascular TOS presents with edema and cyanosis of the upper limbs with decreased pulses ${ }^{2}$. Although arterial complications arising from the compression of the thoracic outlet are present in $<5 \%$ of the operations carried out by TOS, these complications have great medical importance due to their serious prognostic implications. In 1905, Murphy performed the first successful resection of the cervical rib in a patient with TOS and subclavian artery aneurysm using the supraclavicular approach ${ }^{3}$. Arterial TOS is always associated with bone abnormalities, in the cervical rib, a fibromuscular band, or a fracture. TOS generally affects young patients, with an average age of 36 and predominance in males. This disease can be devastating by threatening the upper limbs. Delay in diagnosis is common, which is frustrating for the patient and can lead to permanent damage ${ }^{4}$.

\section{Case study}

A 40-year-old female patient admitted to the High Specialty Regional Hospital (Hospital Regional de Alta Especialidad) of the Yucatan Peninsula, with a 2-week history of pain, paresthesia, muscle weakness, and coldness in the right upper limb. Physical examination
Correspondence:

*Abraham Ziga-Martínez
E-mail: abrahamziga@gmail.com

Available online: 13-09-2019
Date of reception: 15-11-2017

Date of acceptance: 01-02-2018 DOI: 10.24875/HGMX.M19000015
Rev Med Hosp Gen Mex. 2019;82(3):151-154

www.hospitalgeneral.mx 0185-1063/@ 2018 Sociedad Médica del Hospital General de Mexico. Published by Permanyer México SA de CV. This is an open access article under the CC BY-NC-ND license (http://creativecommons.org/licenses/by-nc-nd/4.0/). 


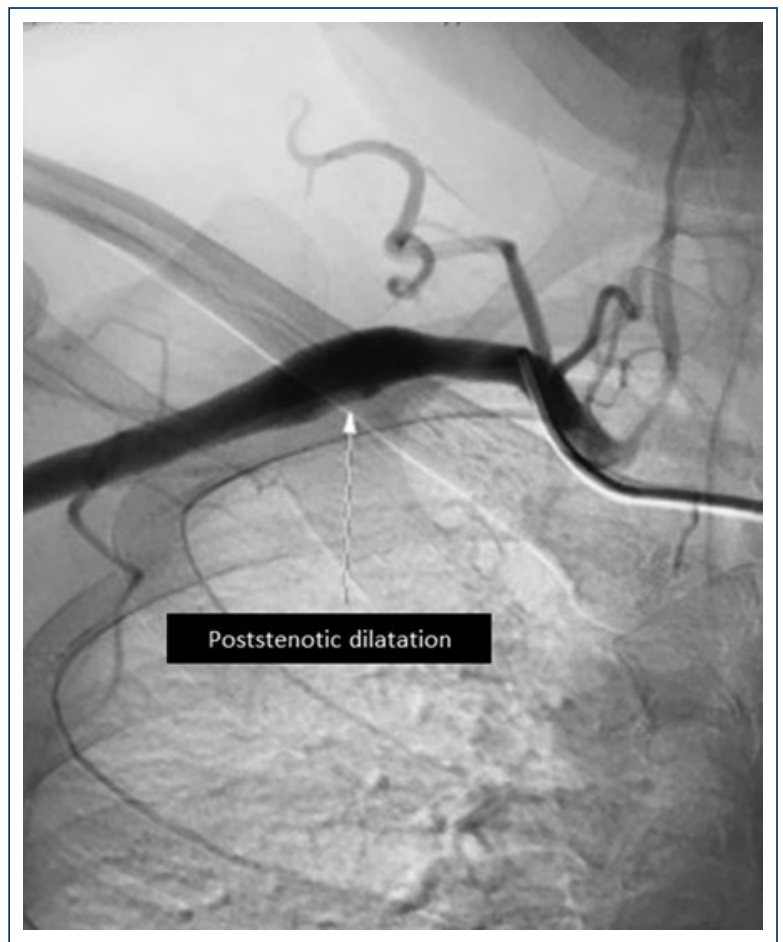

Figure 1. Post-stenotic dilatation in the right subclavian artery.

revealed paleness and coldness of the hand, with decreased muscle strength and absence of pulses. Doppler arterial ultrasound was performed, in which brachial artery thrombosis was documented. Hence, the patient underwent emergency surgery, that is, brachial arterial exploration, where an arteriotomy was performed to obtain an organized thrombus. Embolectomy was performed with a Fogarty catheter, extracting organized thrombi, subsequently obtaining flows. When the operation was completed, weak pulses were obtained in the brachial, radial, and ulnar artery. On the $1^{\text {st }}$ post-operative day, it was decided to perform arteriography of the supra-aortic trunks and thoracic limbs due to high suspicions of TOS, due to the patient's age and as risk factors for arterial thromboembolism were not detected. The arteriography shows dilatation in the subclavian artery posterior to the compression site and severe stenosis, as well as cervical rib (Figs. 1 and 2). Resection of the first cervical rib was performed by the right supraclavicular approach, with post-stenotic dilatation of the right subclavian artery in the transoperative period (Figs. 3 and 4). The patient was discharged on the $2^{\text {nd }}$ post-operative day without complications.

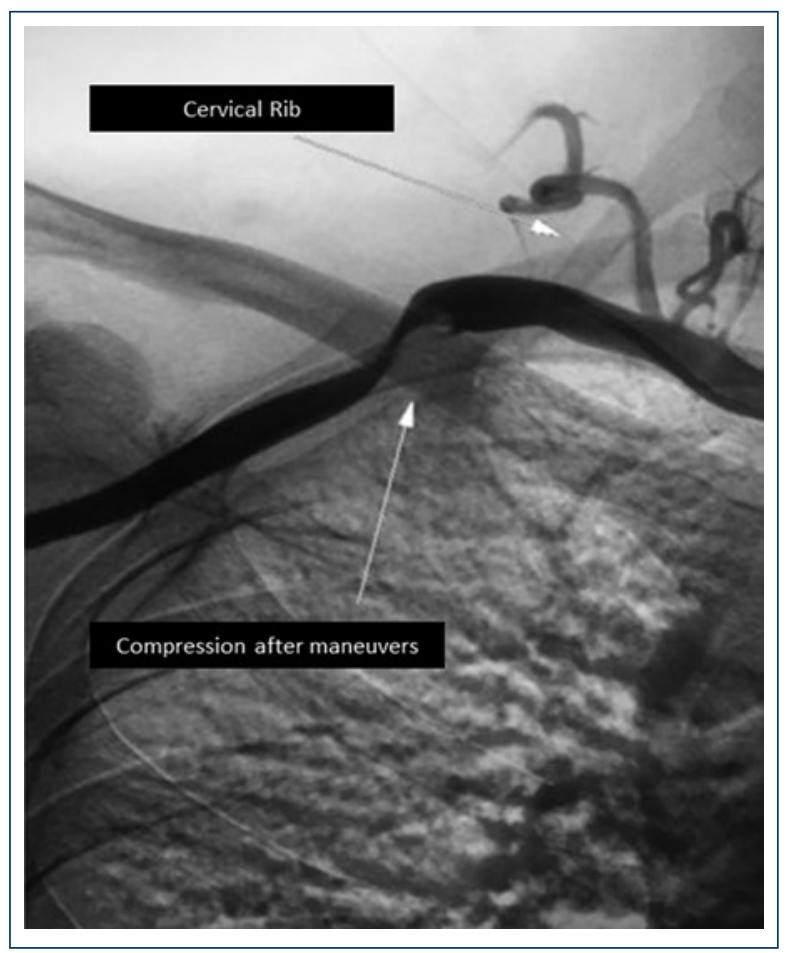

Figure 2. Severe stenosis of the right subclavian artery with abduction of the arm at $45^{\circ}$ (lower arrow) and cervical rib (upper arrow) is observed.

\section{Discussion}

Patients with arterial symptoms constitute only a small fraction of $<5 \%$ of the entire population of patients with neurovascular compression in the thoracic outlet region. TOS is frequently caused by congenital or acquired abnormalities. Regarding patients with arterial TOS, $88 \%$ have bone abnormalities and congenital bone abnormalities are the most frequent cause. The cervical rib is present in most patients in arterial TOS complications ${ }^{5}$. Long-term compression in the subclavian artery leads to various arterial lesions including stenosis with or without mural thrombus, post-stenotic dilatation, and post-stenotic aneurysms. These chronic changes carry risks of complications such as embolism of the arm or hand, leading to acute ischemia of the limb or digital gangrene $^{5,6}$. A simple X-ray of the neck and thorax with a view of the cervical or thoracic spine often shows bone anomalies that can help in the TOS diagnosis. However, the negative predictive value of a simple $X$-ray is debatable, and in almost all cases, a definitive diagnostic image is required to establish or rule out a TOS diagnosis. Arteriography or venography has traditionally been considered the gold standard diagnostic test for vascular TOS, as it provides information on 


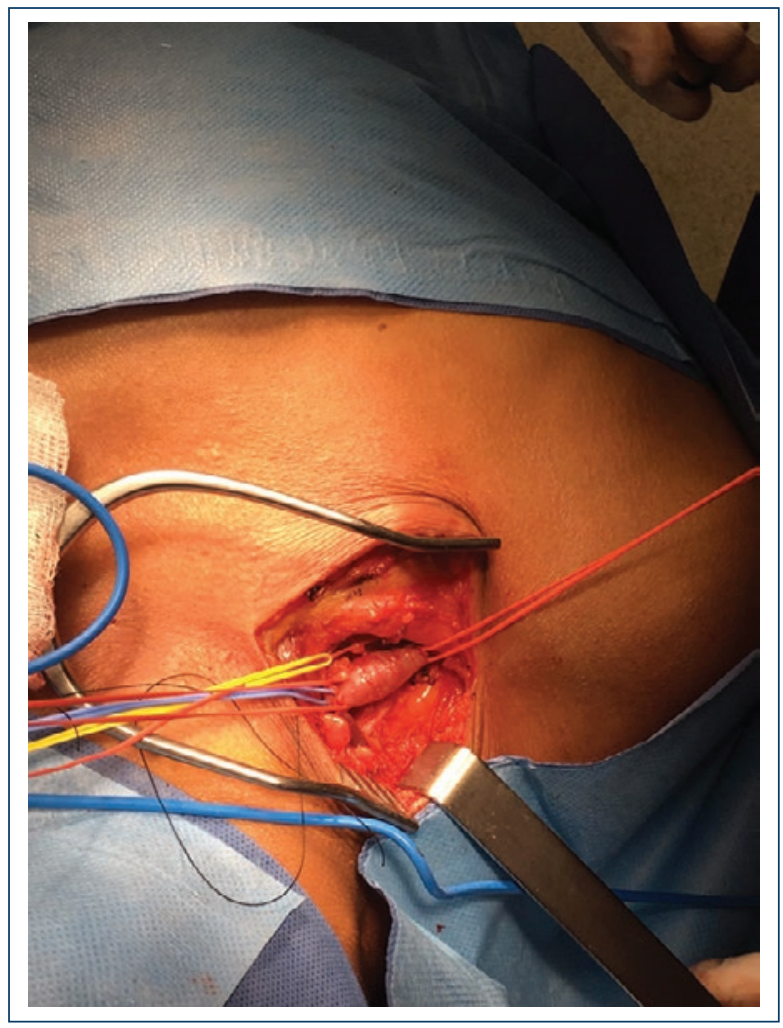

Figure 3. Post-stenotic dilatation of the right subclavian artery seen by the right supraclavicular approach.

the exact location and nature of the vascular compression. However, due to being invasive and the lack of visualization of the surrounding structures, it is only reserved for guidance in interventional procedures. Computed tomography (CT) with contrast and magnetic resonance imaging (MRI) are widely available, reliable, and reproducible modalities used to establish the diagnosis of vascular TOS. CT scans provide information about the vasculature and relationship with adjacent bone structures; MRI is more effective in the representation of accessory muscles, muscle hypertrophy, and fibrous bands. The American College of Radiology recommends CT scans and MRI to establish the diagnosis of $\mathrm{TOS}^{5}$. Surgical treatment is required for patients with symptoms and evidence of arterial complications such as intimal damage, mural thrombus, embolization, post-stenotic dilatation, or aneurysm formation. The appropriate surgical strategy is guided by three main principles: decompression, arterial resection, and distal revascularization. There are two main surgical approaches for TOS decompression: transaxillary and supraclavicular. The main advantage of the transaxillary approach is that it provides a complete visualization of the first rib for its

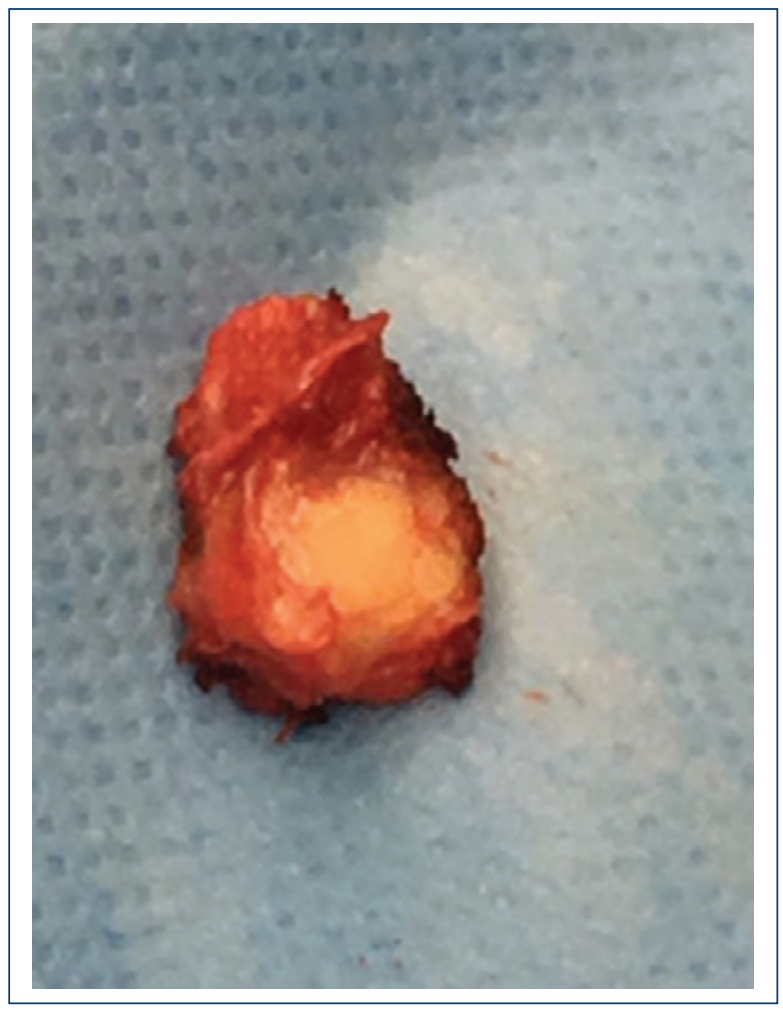

Figure 4. Surgical specimen of cervical rib removed by the right supraclavicular approach, on the day after thrombectomy.

resection and presents a minimal risk of injury to the neurovascular structures. This approach is widely used for neurogenic TOS, as it is not suitable for vascular reconstruction. The supraclavicular approach is preferable for arterial TOS because it allows for the resection of a cervical rib and first rib, fibromuscular structures, and vascular reconstruction ${ }^{3}$.

\section{Conclusion}

Arterial TOS is a rare and relatively uncommon cause of upper limb ischemia. Its complications threaten the life and viability of the limb. Arterial TOS can present itself with symptoms of ischemia related to stress or emboli, typically in the context of an aneurysm with partial thrombosis. Bone abnormalities, such as a cervical rib, a prominent transverse process in the seventh cervical vertebra $(\mathrm{C} 7)$, or a prominent bony callus after a clavicular lesion, are typically associated with this compressive syndrome. Embolization may require urgent treatment with thrombolysis or surgical embolectomy. The supraclavicular approach is usually preferred when arterial reconstruction is indicated for better access to the subclavian artery. 


\section{Conflicts of interest}

The authors declare that there are no conflicts of interest.

\section{Ethical disclosures}

Protection of human and animal subjects. The authors declare that no experiments were performed on humans or animals for this study.

Confidentiality of data. The authors declare that they have followed the protocols of their work center on the publication of patient data.

Right to privacy and informed consent. The authors have obtained the written informed consent of the patients or subjects mentioned in the article. The corresponding author is in possession of this document.

\section{References}

1. Watson LA, Pizzari T, Balster S. Thoracic outlet syndrome part 1: clinical manifestations, differentiation and treatment pathways. Man Ther. 2009;14:586-95.

2. Hussain MA, Aljabri B, Al-Omran M. Vascular thoracic outlet syndrome. Semin Thorac Cardiovasc Surg. 2016;28:151-7.

3. Davidović LB, Koncar IB, Pejkić SD, Kuzmanović IB. Arterial complications of thoracic outlet syndrome. Am Surg. 2009;75:235-9.

4. Thompson JF, Jannsen F. Thoracic outlet syndromes. $\mathrm{Br} J$ Surg 1996;83:435-6.

5. Vemuri C, McLaughlin LN, Abuirqeba AA, Thompson RW. Clinical presentation and management of arterial thoracic outlet syndrome. $\mathrm{J}$ Vasc Surg. 2017;65:1429-39.

6. Illig KA, Donahue D, Duncan A, Freischlag J, Gelabert H, Johansen K et al. Reporting standards of the society for vascular surgery for thoracic outlet syndrome. J Vasc Surg. 2016;64:e23-35. 